The Dysexecutive Questionnaire Revised (DEX-R): an extended measure of everyday dysexecutive problems after acquired brain injury

Simblett, Sara Katherine 1, 2, 3, Ring, Howard ${ }^{1,2,4}$, and Bateman, Andrew 1, 2, 5, 6

${ }^{1}$ Department of Psychiatry, University of Cambridge, UK.

2 National Institute for Health Research (NIHR) Collaborations for Leadership in Applied Health Research and Care (CLAHRC) East at Cambridgeshire and Peterborough NHS Trust, UK.

3 Department of Psychology, Institute of Psychiatry, King's College London, UK.

${ }^{4}$ Cambridgeshire and Peterborough NHS Foundation Trust, UK.

5 Oliver Zangwill Centre for Neuropsychological Rehabilitation, Ely, UK.

6 Cambridgeshire Community Services NHS Trust, UK. 


\section{Acknowledgements}

This research was funded by the National Institute for Health Research (NIHR) Collaboration for Leadership in Applied Health Research and Care East of England at Cambridgeshire and Peterborough NHS Foundation Trust as part of a PhD awarded to the first author, Dr Sara Simblett. The views expressed are those of the author(s) and not necessarily those of the NHS, the NIHR or the Department of Health. We are grateful to Anglia Stroke and Heart Network, the Oliver Zangwill Centre for Neuropsychological Rehabilitation, Ely, UK, and Cambridgeshire Community Services Neurorehabilitation Business Unit who provided extra support in the collection of data for this research. 


\title{
The Dysexecutive Questionnaire Revised (DEX-R): an extended measure of everyday problems with executive functioning after acquired brain injury
}

\begin{abstract}
The Dysexecutive questionnaire (DEX) is a tool for measuring everyday problems with dysexecutive problems. This study investigated the psychometric properties of a revised version of the measure (DEX-R); a comprehensive tool, grounded in current theoretical conceptualisations of frontal lobe function and dysexecutive problems. The aim was to improve measurement of dysexecutive problems following acquired brain injury (ABI). Responses to the DEX-R were collected from 136 men and women who had experienced an ABI (the majority of whom had experienced a stroke or sub-arachnoid haemorrhage) and where possible, one of their carers or family members $(n=71)$, who acted as an informant. Rasch analysis techniques were employed to explore the psychometric properties of four newly developed, theoretically distinct subscales based on Stuss et al.'s (2007) model of 'frontal lobe function' and to evaluate the comparative validity and reliability of self and informant ratings of these four subscales. The newly developed subscales were well targeted to the range of dysexecutive problems reported by the current sample and each displayed a good level of internal validity. Both self- and independent-ratings were found to be performing reliably as outcome measures for at least a group-level. This new version of the tool could help guide selection of interventions for different types of 'dysexecutive' problems and provide accurate measurement in neurorehabilitation services.
\end{abstract}

Keywords: Frontal Lobe Functions, Dysexecutive Syndrome, Dysexecutive Questionnaire, Psychometric Properties, Rasch Analysis, Acquired Brain Injury 


\section{Introduction}

The Dysexecutive syndrome is a very broad term used to capture a range of interacting problems with high-level cognitive, emotional and behavioural abilities. These functions are often relied upon to adapt and respond appropriately to the environment, as well as prioritise and maintain focus on goals. Damage to the frontal lobes, specifically, the prefrontal cortex (PFC), have been found to play an important, albeit not an exclusive, role in dysexecutive problems (see meta-analysis by Alvarez \& Emory, 2006). Indeed, the impairments associated with dysexecutive problems previously came to be known as 'frontal lobe syndrome'. Research suggests that the concept of 'frontal lobe functions' can be sub-divided into several domain general functions (Cicerone, Levin, Malec, Stuss, \& Whyte, 2006; Stuss, 2007). These include 'Executive cognitive functions', defined as high-level cognitive functions involved in the 'control and direction of lower-level, more automatic functions', e.g. functions associated with memory, language, perception, attention etc.; 'Behavioural-emotional self-regulatory functions', which rely on actions driven by emotional responses required in 'situations where cognitive analysis, habit or environmental cues are not sufficient to determine the most adaptive response'; and 'Activation regulating functions' (sometimes referred to as 'Energisation regulating functions') that underlie the ability to 'generate and maintain actions or mental processes' (Cicerone et al., 2006; Stuss, 2007). Stuss and colleagues described a fourth domain, 'Metacognitive functions', that are thought to be involved in the integration of the three areas of frontal lobe function previously defined, and are necessary for self-awareness, social cognition and autonoetic consciousness. Although in Stuss's model is concerned with sub-dividing 'frontal lobe functions' the domains can also be conceptualised as different processes involved in the control and direction of cognition, emotion and behaviour, and contribute to differences in presentations of dysexecutive problems. 
A significant minority of people who experience an acquired brain injury have been found to experience dysexecutive problems. Indeed, it is estimated that $40 \%$ of people who experience a stroke are left with impairments particularly associated with 'Executive cognitive functions' (Hoffmann \& Schmitt, 2006; Pohjasvaara et al., 2002). People who experience other types of ABI, such as Traumatic Brain Injuries (TBI), are even more vulnerable to experiencing dysexecutive problems due to common disruptions frontal-subcortical brain systems (McDonald, Flashman, \& Saykin, 2002). Dysexecutive problems can severely impact on a person's ability to function independently and productively in everyday life (Hanks, Rapport, Millis, \& Deshpande, 1999). They have also been shown to reduce people's ability to choose appropriate and adaptive coping strategies in response to stress (Gyurak et al., 2009; Gyurak, Goodkind, Kramer, Miller, \& Levenson, 2011; Krpan, Levine, Stuss, \& Dawson, 2007) that are conducive to good mental wellbeing (Côté, Gyurak, \& Levenson, 2010). This may be one reason why some dysexecutive problems have been found to be associated with symptoms of depression and anxiety following ABI (Barker-Collo, 2007; Pohjasvaara et al., 2002; Vataja et al., 2005).

Given the major impact that dysexecutive problems can have on people's lives following ABI it is important that neurorehabilitation services seek to identify those in need of support and help them cope with these difficulties. However, as already highlighted, dysexecutive problems can present in differently and relate to differences in the underlying brain regions affected by the injury (Stuss, 2007) and probably require different forms of support (see review by Cicerone, Levin, Malec, Stuss \& Whyte, 2007). Measurement tools that help to accurately differentiate between different types of executive impairments could be used to help guide rehabilitation and measure change following targeted intervention. 
The Dysexecutive questionnaire (DEX; Burgess, Alderman, Evans, Wilson, \& Emslie, 1996) has been introduced as one such tool (Simblett \& Bateman, 2010). A valid and reliable three dimensional structure for the DEX has been proposed (Simblett et al., 2012; Simblett \& Bateman, 2010), that is in keeping with current theoretical conceptualisations of domain general processes associated with 'frontal lobe' or executive functions (Cicerone et al., 2006; Stuss, 2007). However, previous research carried out by Simblett and Bateman (2011) on the psychometric properties of the DEX has highlighted some ways in which the measure could be improved. First, it was suggested that some of the items could be rephrased to clarify the intended meaning, with the aim of reducing likelihood of variability in interpretation and responding. This included removing any unnecessary additional quantifiers such as the word "sometimes" in item 3 (confabulation: "occasionally", "sometimes" or "fairly often". . . "I [sometimes] talk about events or details that never actually happened, but I believe did happen"). Doubled-barrelled questions were separated into two individual items; for example item 4 (planning) was divided into the two items: "I have difficulty thinking ahead" and "I have difficulty planning for the future". For further discussion of these changes see Simblett and Bateman (2011).

Second, it was also noted that the DEX was heavily weighted to measuring ‘Behavioural-emotional Self-regulatory' functions (in accordance with Stuss et al, 2007, model of 'frontal lobe function'; see introduction for a definition of terms) and that there was scope to develop the tool into a broader measure that included equal an emphasis of 'executive cognitive' and 'Metacognitive' domains. Furthermore, it was hypothesised that the 'Behavioural-emotional Self-regulatory' subscale included items that could, arguably, be measuring a fourth domain, 'Activation Regulating' functions, as defined by Stuss, and that separating the items into two sets might improve the validity and reliability of the tool. Further 
items weighted towards measurement of 'Activation Regulating' functions were added to draw out existing items within the 'Behavioural-emotional Self-regulatory' subscale of the original DEX that were more likely to fit within the former category of dysexecutive problems.

This study sought to evaluate the impact of several changes to the DEX following on from previous recommendations made by Simblett and Bateman (2010), with the aim of refining the psychometric properties of the DEX and developing a more comprehensive tool that could provide an improved measure or bank of items for measuring problems with executive functioning following ABI: the DEX-R.

\section{Methods}

\section{Participants}

Participants aged 18 years or older (ranging from 19 to 90 years old), diagnosed with a non-progressive brain injury, the majority of whom had experienced a non-traumatic brain injury (nTBI) such as a cerebrovascular accident (CVA, including ischemic and haemorrhagic strokes), who were able to provide informed consent were recruited through community neurorehabilitation services. All participants demonstrated adequate communication skills to respond to the questionnaire with the support of a researcher. Demographic characteristics of the sample as well as information about the nature of their brain injury, including time post-injury and their current level of functioning ability as measured by the Nottingham Extended Activities of Daily Living Scale (EADLS; Lincoln \& Gladman, 1992; Nouri \& Lincoln, 1987) was gathered where possible (this information was not available from the national specialist service that provided a proportion of the data), the details of which can be found in Table 1. Higher scores indicate a higher degree of functional independence. Where 
possible, a carer or family member of each person who had experienced an ABI was also approached and asked if they would consent to completing an informant-rating version of the DEX-R.

\section{Measure}

The DEX (Burgess, Alderman, Wilson, Evans, \& Emslie, 1996) is a 20-item scale designed specifically to measure everyday manifestations of dysexecutive problems after acquired brain injury. Respondents are asked to rate on a 5-point Likert scale how often they experience certain difficulties associated with control and direction of cognition, emotion and behaviour (e.g. with planning, impulsivity, motivation etc.). The DEX questionnaire is available in two different formats, a self-rating version for people with ABI to complete (DEX-S) and an independent-rating version for someone close to that person to complete on their behalf (DEX-I). Both self-rating and independent-rating versions of the original DEX have previously been validated as measurement tools within an ABI population. They have been found to be sensitive tools with good psychometric properties (Bennett, Ong, \& Ponsford, 2005; Bodenburg \& Dopslaff, 2008; Boelen, Spikman, Rietveld, \& Fasotti, 2009; Chan \& Bode, 2008; Simblett et al., 2012; Simblett \& Bateman, 2010).

Following on from Simblett and Bateman's (2010) research on the psychometric properties of the DEX as a measure of dysexecutive problems following $\mathrm{ABI}$, a number of changes were made to the original DEX based on suggested improvements (see introduction for further 
information). First, some of the items were reworded or divided into two separate questions to improve the clarity of items in the measure. Second, a further 14 items were included in the new version of the measure with the aim of broadening the tool's range of measurement. These new items were developed to fit within one of the theoretical domains of 'frontal lobe function' as defined by Stuss but further scientific justification was sought for the content of each individual item from sources that had demonstrated a link between a specific behaviour or cognitive ability and a process involved in executive functioning or with a specific region of the prefrontal cortex. The new items included in the measure are shown in Table 2. Table 3 displays a list of all items in the revised version of the DEX, which will be referred to as the DEX-R, as well as the theoretical domain that each was predicted to belong to. As with the DEX, two versions of the DEX-R were created to provide self-ratings (DEX-R-S) as well as independent-ratings (DEX-R-I).

$* * * * * * * * * * * * * * * * * * * * * * * * * * * * *$ Table 2 about here $* * * * * * * * * * * * * * * * * * * * * * * * * * * * * *$

$* * * * * * * * * * * * * * * * * * * * * * * * * * * * *$ Table 3 about here $* * * * * * * * * * * * * * * * * * * * * * * * * * * * * *$

\section{Data analysis}

Two separate Rasch analyses using the software RUMM2020 (Andrich, Sheridan, \& Lou, 2005) were carried out on responses to self-rating and independent-rating versions of the DEX-R. Missing responses to individual items were retained in each analysis and estimates of probable rating were imputed as part of the Rasch analyses (Granger, 2008). The aim of these analyses was as follows: 
1. To develop four theoretically distinct subscales. More specifically, to explore whether inclusion of additional items theorised to measure the 'Activation' domain enabled separation of items into two subscales measuring distinctive processes involved in 'emotional-behavioural self-regulation' and 'Activation' as defined by Stuss (2007), in addition to subscales measuring 'Metacognition' and 'Executive Cognition'. An exploratory factor analysis on the residuals for each item, i.e. the variance not explained by the Rasch model (also known as a Rasch factor analysis), was performed on all items theorised to measure either 'Activation' or 'Behaviour-emotional Self-regulatory' functions to help separate these items into two groups. Items with highest loadings on the first principle components were grouped together and items (in order of decreasing loadings) were added until the subscale was no longer unidimensional (following the method proposed by Smith, 2002). The remaining items were then grouped together and tested to see if they made up a separate unidimensional construct.

2. To broaden the range of measurement of the tool. In particular, to explore whether additional items included in the 'Metacognition' and 'Executive Cognition' subscales broaden the range of measurement and could therefore be used to assess a wider degree of impairment in these two processes. This was established by comparing the range of log-odd units (logits) measured by the new subscales as compared to those measured by the equivalent subscales from the original DEX. As part of this, the targeting of the items to the sample was examined to explore the proportion of people whose ability level was captured by each subscale as an indication of the appropriateness of each as a separate measure of dysexecutive problems. 
3. To investigate the measurement properties of the new subscales. An important aim was to establish whether all subscales met the assumptions of the Rasch measurement model and could therefore be considered to possess interval-level measurement properties. The procedure undertaken by Simblett and Bateman in their previous analyses of the original DEX (Simblett and Bateman 2010, Simblett, Badham et al. 2012) that followed guidelines written by Smith, Linacre, and Smith (2006) and Tennant and Conaghan (2007), was employed to establish whether each subscale met the assumptions of the Rasch measurement model. Chi-square statistics, with bonferroni corrections for multiple comparisons, were used to indicate goodness of fit to the Rasch model. If the subscales did not meet the assumptions of the Rasch model, further investigations were carried out exploring potential threats to construct validity. This included exploring whether the subscale was multidimensional and whether any individual items misfit the Rasch model (displayed fit residuals $>2.5$ or $<-2.5$ ) or presented with disordered thresholds (the probability of endorsing response categories did not correspond to the progression of the underlying metric), local dependency (showed correlations between the residuals of two items > 0.3), or differential item functioning (DIF; indicative of response bias) on the basis of individual characteristics such as gender, age and person rating the scale (significant statistical differences between groups for a single item). The fit of responses from individual participants in the sample was also examined to establish if anyone had provided responses that were unusually erratic (displayed fit residuals > 2.5) and potentially unreliable sources of information. If problems were uncovered an iterative process of rescoring and removal of items and/or persons were perform and further tests were carried out to investigate how these changes impacted on the overall fit of the data to the Rasch Model Reliability of subscales was assessed based on the person separation index (PSI). Tennant and Conaghan (2007) suggest this is equivalent to the interpretation of Cronbach's 
alpha statistic (Cronbach, 1951), with a value greater than 0.7 indicating suitability for group-level use and a value greater than 0.9 indicating suitability for individual-level use (this index is equivalent to the WINSTEPS item separation ratio, where values greater than 1.5 would be required for group use).

4. To establish whether self- and informant-ratings are equally valid and reliable. All previous analyses were performed on responses to the self-rating version of the DEX-R. However, a further aim was to explore whether subscales displayed a similar degree of validity and reliability when rated by participants who had experienced an acquired brain injury as compared to a family member or carer acting as an informant. A comparison of chi-square statistics used to establish goodness of fit to the Rasch measurement model and the PSI for self- and informant-ratings of the subscales was used to guide conclusions in relation to this aim.

\section{Results}

A total of 137 responses to the self-rating version of the DEX-R were collected, along with 71 responses to the independent-rating version of the DEX-R. The median age of respondents to the DEX-R-S was 67 years old (IQR: 56-76). Of these, $84.6 \%$ and $84.5 \%$ of questionnaires were returned complete for the self- and independent-rating versions, respectively. There was a relatively even spread of missing responses across the items in the measure. The percentage of missing responses was no greater than $5 \%$ for all items in the self-rating version and only one item (item 30 - Restlessness) in the independent-rating version exceeded this value, with $5.6 \%$ of respondents leaving this item unanswered. 
Examination of the content and psychometric properties of the proposed subscales. Examination of the subscales of the DEX-R proposed (see Table 3) using self-rated responses revealed promising results. However, some modifications were required in order for the subscales to fit the Rasch model. For the 'Executive Cognition' subscale, one of the new items, item 2 - Prospective memory, and responses from three specific participants in the sample displayed misfit to the Rasch model, which suggested that they may be unreliable sources of information. Removal of item 2 and the three respondents meant that the subscale achieved fit to the expectations of the Rasch model (see Table 4, analysis 4 as compared to analysis 3 ).

********************************) Table 4 about here $* * * * * * * * * * * * * * * * * * * * * * * * * * * * * *$

The 'Metacognition' subscale including the additional items displayed good fit to the Rasch model without modification (see Table 4, analysis 1). However, two of the new items (item 14 - meta-worry and item 22 - cognitive control) displayed correlated residuals (i.e. local dependency), indicating that they may be too similar. Removal of item 22 resulted in the greatest improvement in fit to the Rasch model, indicated by change in the item-trait chi-square statistic (see Table 4, analysis 2 for results of this change).

A subscale including all items in the original 'Behavioural-emotional Self-regulation' scale (Simblett \& Bateman, 2010) along with additional items designed to measure the 'Activation' domain, significantly deviated from the expectations of the Rasch model and did not pass the test of unidimensionality (see Table 4, analysis 5). Further investigation by way of an exploratory Rasch factor analysis, revealed that this subset of items could be divided into two 
separate unidimensional subscales, which fitted the Rasch model and displayed a sufficient level of reliability for at least group use (see Table 4, analyses 6 and 8). These are theorised to measure the two domains, 'Behavioural-emotional Self-regulation' and 'Activation', separately. Inspection of residual correlations revealed that two items in the new 'Behavioural-emotional Self-regulation' subscale were correlated (items 16 - Blunted affect1 and 33 - Blunted affect2). The association between these items was not surprising given that only one word differed between the two items. Indeed, item 16 asked if they had difficulty 'showing' emotion and item 33 asked if they had difficulty 'expressing' emotion. The greatest improvement to the item-trait chi-square statistic resulted from the removal of item 33 , therefore, item 16 was kept in the 'Behavioural-emotional Self-regulation' subscale (see Table 4, analysis 7 for results of this change). Investigation of response biases within grouping variables revealed only one significant finding.

The four new subscales appeared to measure a good range of difficulties associated with the four separable domains and were relatively well targeted to the sample (see Figure 1 and Table 5), indicating that a large proportion of the sample endorsed at least some dysexecutive problems. The items added to the subscales increased the range of measurement to represent a more diverse sample of abilities or impairments within the four domains of functioning. The individual location values for each item within the four subscales, summed across all thresholds, are displayed in Table 6, giving an idea of the types of problems most commonly reported for each of the four domains.

********************************) Figure 1 about here $* * * * * * * * * * * * * * * * * * * * * * * * * * * * * *$ 
Comparison of self and independent responses to the proposed subscales. Analysis of independent ratings on the DEX-R confirmed the findings for the self-rated DEX-R (see Table 7), suggesting that they are also valid, reliable and appropriately targeted tools for measuring executive functioning following ABI. However, exploration of differential item functioning did highlight one potential difference between the two samples of raters. Independent raters were more likely than self raters to endorse item 30 (restlessness) but this discrepancy only existed when the person who had experienced the brain injury demonstrate moderate problems with 'Activation' overall (for the $2^{\text {nd }}$ class interval, only; see Figure 4). From inspection of Figure 2, it would appear that the self-ratings on this item conforms better to the pattern expected by the Rasch model and thus, suggests that perhaps self-ratings for this item show a slightly greater degree of validity.

*******************************) Table 7 about here $* * * * * * * * * * * * * * * * * * * * * * * * * * * * * *$

*******************************) Figure 2 about here $* * * * * * * * * * * * * * * * * * * * * * * * * * * * * *$

\section{Discussion}

This paper describes a process of modifying the DEX to create a revised version of the questionnaire, with the aim of improving the measurement of dysexecutive problems following 
ABI. This involved rephrasing some items from the original DEX that have been found in previous research to provide unreliable estimates of functioning (Simblett \& Bateman, 2010) as well as introducing several new items with the intention of broadening the scope of the measure based recent developments in the theoretical conceptualisation of the 'dysexecutive syndrome' or 'frontal lobe' functioning.

Analysis of responses to the questionnaire at an item-level based on Rasch analysis techniques, showed that some of the new items introduced to the DEX measured concepts that were too highly correlated with one another or provided unreliable information, and were therefore discarded from the DEX-R. Following on from these post-hoc modifications, four psychometrically distinct subscales have been created that are hypothesised to correspond to the four domains of frontal lobe functions identified by Stuss (2007). Some problems associated with the interpretation of response categories remained, which indicates that further work may be needed to simplify the measure and reduce the ambiguity of response categories. This may be especially important if measures are used to assess people who have cognitive impairments and/or some communication difficulties that could further impede interpretation and understanding. It is possible that the number of response categories should vary between items. Indeed, not all items were found to have disordered thresholds. For some items, it may only make sense to respond either 'yes' or 'no', while for others, further gradations are possible and provide a richer level of information. However, items included in the current version of the measure were not rescored due to a small sample size and the potential for unstable solutions. The modifications described in this paper expand upon the original DEX to provide a more comprehensive measure of difficulties with everyday activities that could result from dysexecutive problems that interfere with the control of cognitive, behavioural and emotional processes. The new items added to the measure mean that a broader range of everyday 
difficulties can be measured with the tool, specifically with regards to executive cognitive functions that allow people to think flexibly and metacognitive functions that enable a person to reflect on their own thoughts, emotions and behaviour. In addition, problems associated with emotional-behavioural self-regulatory functions, e.g. poor control of actions due to difficulties regulating emotions and behaviour, can be measured separately to those involved in 'Activation', e.g. poor control of actions due to low levels of general arousal. It is suggested that scores on the four individual subscales of the measure could be used to identify specific areas of weakness associated with slightly different types of executive impairments following ABI and help to tailor treatment interventions to the needs of the individual.

A good response rate to all items on the DEX-R, including the additional items, was found and all four subscales were well targeted to the sample. This suggests that the questions were acceptable and appropriate for most of the respondents, the majority of whom had experienced a stroke within the last five years (average time post stroke was two years) and were no longer receiving rehabilitative support. This finding extended to independent responses to the DEX-R, provided by carers/family members.

In addition to finding that the DEX-R is suitable measure of dysexecutive problems following brain injury, the results potentially highlight an area of unmet need. Indeed, many of the participants were still experiencing dysexecutive problems in everyday life despite having experienced their stroke years previously and no longer receiving any rehabilitation. Thus, it is suggestive that survivors of ABI may benefit from further support with 'executive' processes. Some of the most frequently endorsed problems were with finding the right words to say; doing two activities at once; keeping several pieces of information in mind at once; telling people that they disagreed; and having confidence in one's own cognitive abilities. Arguably, these are challenges that exist within a spectrum of 'normal' functioning and may not indicate 
dysexecutive problems as a result of organic damage to the brain. However, this information may be useful for guiding provision of extra support for dysexecutive problems following ABI if these difficulties exist and are impacting on their ability to function in everyday life.

All but one of the items (item 30 - restlessness) was answered in a similar manner regardless of whether ratings were provided by the group of people who had experienced the ABI or a carer/family member. The reason for the response bias found for item 30 is not clear but, given that self-reporting was found to be more reliable for this item, it may relate to the ease at which problems could be observed or awareness of this problem. Indeed, difficulties with awareness have been noted to be a common symptom associated with the dysexecutive syndrome and discrepancy between the magnitude of self and independent ratings on the DEX has in the past been used as a means of gauging severity of problems with awareness (Burgess et al, 1998; Hart et al., 2005; McGuire et al., 2014). Alternatively, the discrepancy in reliability might relate to a greater degree of missing data from independent raters as compared to self raters for this item. Item 30 (restlessness) was retained in the 'Activation' subscale but it is worth noting, when interpreting scores on this measure that the perspective of the person rating this item could affect responding. Overall, the reliability indices indicated a level of reliability suitable for monitoring outcomes across a group or service for all subscales using either self or independent rating versions of the DEX-R. In addition, the executive cognitive functions subscale clearly demonstrated psychometric properties that make it reliable enough to monitor change within a single individual. Other subscales could be used for measuring change within a single individual across a therapeutic intervention but it is important to note that there may be a greater degree of error in prediction. Although reliability indices were slightly different when the original DEX was rated by independent observers, as compared to the person experiencing the problems themselves (see Simblett, Badham, Greening, Ring, Adlam \& Bateman, 2012), 
reliability indices were similar between independent- and self-ratings on the DEX-R.

Further research is needed to examine the external validity of the DEX-R, for instance, if responses to the subscales of the DEX-R correlate with other measures of the four domains of 'frontal lobe function', including behavioural as well as other self-report measures and the sensitivity and specificity for classifying impairments into different functional domains. Given that this sample included only a small number of participants who had experienced a TBI $(n=8)$, further validation that includes a broader range of ABI aetiologies may be necessary to confirm appropriate use with this clinical group. This may provide an opportunity to hone inclusion of items in the existing measure and create a shorter version of the tool for use in neurorehabilitation services. Another useful addition to the literature would also be to investigate if the DEX-R is an appropriate measure for use in other clinical populations that have also been found to experience dysexecutive problems (e.g. Loschiavo-Alvares et al., 2013).

However, the findings reported in this paper demonstrate that both the self- and independent-rating versions of the DEX-R have good internal validity and reliability as a measure of everyday dysexecutive problems following ABI. Responses to the DEX-R should be considered in terms of four separate subscales, measuring four domain general types of dysexecutive problems. This is in keeping with the structure of the measure initially hypothesised following on from the theoretical conceptualisations of frontal lobe function proposed by Stuss (2007) that builds on an extensive literature base investigating the effects of lesions to circumscribed regions of the prefrontal cortex. There is potential for this new version of the measure to help prioritise and guide selection of interventions for different types of problems associated with the overarching term 'dysexecutive syndrome' and provide accurate estimations of treatment efficacy for neurorehabilitation services specialising in acquired brain 
The DEX-R for acquired brain injury

injury.

\section{References}

Alvarez, J.A., \& Emory, E. (2006). Executive function and the frontal lobes: a meta-analytic 
review. Neuropsychological Review, 16(1), 17-42.

Andrich, D., Sheridan, B., \& Lou, G. (2005). Rumm2020 (Version Version 4 for Windows).

Barker-Collo, Suzanne L. (2007). Depression and anxiety 3 months post stroke: Prevalence and correlates. Archives of Clinical Neuropsychology, 22(4), 519-531.

Bennett, P.C., Ong, B., \& Ponsford, J.L. (2005). Measuring executive dysfunction in an acute rehabilitation setting: Using the dysexecutive questionnaire (DEX). Journal of the International Neuropsychological Society, 11(4), 376-385.

Bodenburg, S., \& Dopslaff, N. (2008). The Dysexecutive Questionnaire Advanced: Item and Test Score Characteristics, 4-Factor Solution, and Severity Classification. The Journal of Nervous and Mental Disease, 196(1), 75-78.

Boelen, D.H.E., Spikman, J.M., Rietveld, A.C.M., \& Fasotti, L. (2009). Executive dysfunction in chronic brain-injured patients: Assessment in outpatient rehabilitation. Neuropsychological Rehabilitation, 19(5), 625-644.

Burgess, P.W., Alderman, N., Evans, J.J., Wilson, B.A., \& Emslie, H. (1996). The dysexecutive questionnaire. In B. Wilson, N. Alderman, P. Burgess, H. Emslie \& J. Evans (Eds.), Behavioral Assessment of the Dysexecutive Syndrome. Bury St Edmunds, UK: Thames Valley Test Company.

Burgess, P.W., Alderman, N., Wilson, B.A., Evans, J.J., \& Emslie, H. (1996). The dysexecutive questionnaire. UK: Psychological Corporation.

Burgess, P.W., \& Shallice, T. (1996). Response suppression, initiation and strategy use following frontal lobe lesions. Neuropsychologia, 34(4), 263-272.

Burgess, P. W., Alderman, N., Evans, J., Emslie, H., \& Wilson, B. A. (1998). The ecological validity of tests of executive function. Journal of the International Neuropsychological Society, 4(06), 547-558.

Buss, A.H., \& Perry, M. (1992). The Aggression Questionnaire. Journal of Personality and 
Social Psychology, 63(3), 452-459.

Cartwright-Hatton, S., \& Wells, A. (1997). Beliefs about Worry and Intrusions: The Meta-Cognitions Questionnaire and its Correlates. Journal of Anxiety Disorders, 11(3), 279-296.

Chan, R.C.K., \& Bode, R.K. (2008). Analysis of patient and proxy ratings on the Dysexecutive Questionnaire: an application of Rasch analysis. Journal of Neurology, Neurosurgery \& Psychiatry, 79(1), 86-88.

Cicerone, K., Levin, H., Malec, J., Stuss, D., \& Whyte, J. (2006). Cognitive Rehabilitation Interventions for Executive Function: Moving from Bench to Bedside in Patients with Traumatic Brain Injury. Journal of Cognitive Neuroscience, 18(7), 1212-1222.

Côté, S., Gyurak, A., \& Levenson, R.W. (2010). The ability to regulate emotion is associated with greater well-being, income, and socioeconomic status. Emotion, 10(6), 923-933.

Cronbach, L. (1951). Coefficient alpha and the internal structure of tests. Psychometrika, 16(3), 297-334.

Devinsky, O., Morrell, M.J., \& Vogt, B.A. (1995). Contributions of anterior cingulate cortex to behaviour. Brain, 118(1), 279-306.

Granger, C. (2008). Rasch Analysis is Important to Understand and Use for Measurement. Rasch Measurement Transactions, 21 (3), 1122-1123.

Groot, Y.C.T., Wilson, B.A., Evans, J.J., \& Watson, P. (2002). Prospective memory functioning in people with and without brain injury. Journal of the International Neuropsychological Society, 8(05), 645-654.

Gyurak, A., Goodkind, M., Madan, A., Kramer, J., Miller, B., \& Levenson, R.W. (2009). Do tests of executive functioning predict ability to downregulate emotions spontaneously and when instructed to suppress? Cognitive, Affective, \& Behavioral Neuroscience, $9(2), 144-152$. 
Gyurak, A., Goodkind, M.S., Kramer, J.H., Miller, B.L., \& Levenson, R.W. (2011). Executive functions and the down-regulation and up-regulation of emotion. Cognition \& Emotion, 26(1), 103-118.

Hanks, R.A., Rapport, L.J., Millis, S.R., \& Deshpande, S.A. (1999). Measures of executive functioning as predictors of functional ability and social integration in a rehabilitation sample. Archives of Physical Medicine and Rehabilitation, 80(9), 1030-1037.

Hart, T., Whyte, J., Kim, J., \& Vaccaro, M. (2005). Executive Function and Self-awareness of "Real-world" Behavior and Attention Deficits Following Traumatic Brain Injury. The Journal of head trauma rehabilitation, 20(4), 333-347.

Hoffmann, M., \& Schmitt, F. (2006). Metacognition in Stroke: Bedside Assessment and Relation to Location, Size, and Stroke Severity. Cognitive and Behavioral Neurology, $19(2), 85-94$.

Hornak, J., O'Doherty, J., Bramham, J., Rolls, E.T., Morris, R.G., Bullock, P.R., \& Polkey, C.E. (2004). Reward-related Reversal Learning after Surgical Excisions in Orbito-frontal or Dorsolateral Prefrontal Cortex in Humans. Journal of Cognitive Neuroscience, 16(3), 463-478.

Krpan, K.M., Levine, B., Stuss, D., \& Dawson, D.R. (2007). Executive function and coping at one-year post traumatic brain injury. Journal of Clinical and Experimental Neuropsychology, 29(1), 36-46.

Lincoln, N.B., \& Gladman, J.R.F. (1992). The Extended Activities of Daily Living scale: a further validation. Disability and Rehabilitation, 14(1), 41-43.

Loschiavo-Alvares, F.Q., Sediyama, C.Y.N., Vasconcelos, A.G., Neves, F., Corrêa, H., Malloy-Diniz, L.F., \& Bateman, A. (2013). Clinical application of DEX-R for patients with bipolar disorder type I and II. Clinical Neuropsychiatry, 10 (2), 86-94. 
McGuire, B. E., Morrison, T. G., Barker, L. A., Morton, N., McBrinn, J., Caldwell, S., Wilson, C.F., McCann, J., Carton, S., Delargy, M., \& Walsh, J. (2014). Impaired self-awareness after traumatic brain injury: inter-rater reliability and factor structure of the Dysexecutive Questionnaire (DEX) in patients, significant others and clinicians. Frontiers in behavioral neuroscience, $8,352$.

MacDonald, A.W., Cohen, J.D., Stenger, V.A., \& Carter, C.S. (2000). Dissociating the Role of the Dorsolateral Prefrontal and Anterior Cingulate Cortex in Cognitive Control. Science, 288(5472), 1835-1838.

Martin, M., Kliegel, M., \& McDaniel, M.A. (2003). The involvement of executive functions in prospective memory performance of adults. International Journal of Psychology, 38(4), 195-206.

McDonald, Brenna C., Flashman, Laura A., \& Saykin, Andrew J. (2002). Executive dysfunction following traumatic brain injury: Neural substrates and treatment strategies. NeuroRehabilitation, 17(4), 333-344.

Nouri, F.M., \& Lincoln, N.B. (1987). An extended activities of daily living scale for stroke patients. Clinical Rehabilitation, 1(4), 301-305.

Pohjasvaara, T., Leskelä, M., Vataja, R., Kalska, H., Ylikoski, R., Hietanen, M., Leppävuori, A., Kaste, M., \& Erkinjuntti, T. (2002). Post-stroke depression, executive dysfunction and functional outcome. European Journal of Neurology, 9(3), 269-275.

Schwartz, M. (2000). Executive Function Questionnaire (Version 2.34). New Haven, CT Copyright 2000-2007 by Schwartz, M.

Schwartz, M.F., Montgomery, M.W., Buxbaum, L.J., Lee, S.S., Carew, T.G., Coslett, H.B., Ferraro M, Fitzpatrick-DeSalme E, Hart T, \& Mayer, N. (1998). Naturalistic action impairment in closed head injury. Neuropsychology, 12(1), 13-28.

Simblett, S.K., Badham, R., Greening, K., Adlam, A., Ring, H., \& Bateman, A. (2012). 
Validating independent ratings of executive functioning following acquired brain injury using Rasch analysis. Neuropsychological Rehabilitation, 22(6), 874-889.

Simblett, S.K., \& Bateman, A. (2010). Dimensions of the Dysexecutive Questionnaire (DEX) examined using Rasch analysis. Neuropsychological Rehabilitation, 21(1), 1-25.

Smith, E. V. (2002). Detecting and evaluating the impact of multidimensionality using item fit statistics and principal component analysis of residuals. Journal of Applied Measurement, 3(2), 205-231.

Smith, R. M., Linacre, J. M., \& Smith, E. V. (2006). Guidelines for manuscripts. Journal of Applied Measurement.

Stuss, D. (2007). New approaches to prefrontal lobe testing. In B. Miller \& J. Cummings (Eds.), The human frontal lobes: functions and disorders. New York: Guildford Press.

Stuss, D., \& Levine, B. (2002). Adult Clinical Neuropsychology: Lessons from Studies of the Frontal Lobes. Annual Review of Psychology, 53(1), 401-433.

Tennant, A., \& Conaghan, P. G. (2007). The Rasch measurement model in rheumatology: What is it and why use it? When should it be applied, and what should one look for in a Rasch paper? Arthritis Care \& Research, 57(1358-1362), 1358-1362.

Vataja, R., Pohjasvaara, T., Mäntylä, R., Ylikoski, R., Leskelä, M., Kalska, H., Hietanen, M., Juhani Aronen, H., Salonen, O., Kaste, M., Leppävuori, A., \& Erkinjuntti, T. (2005). Depression-Executive Dysfunction Syndrome in Stroke Patients. American Journal of Geriatric Psych, 13(2), 99-107. 